\title{
The reliability of the ankle-brachial index in the Atherosclerosis Risk in Communities (ARIC) study and the NHLBI Family Heart Study (FHS)
}

\author{
Beth D Weatherley ${ }^{1}$, Lloyd E Chambless ${ }^{2}$, Gerardo Heiss*3, Diane J Catellier ${ }^{2}$ \\ and Curtis R Ellison 4
}

\begin{abstract}
Address: ${ }^{1}$ Duke Clinical Research Institute, Duke University Medical Center, Durham, NC, USA, ${ }^{2}$ Department of Biostatistics, School of Public Health, University of North Carolina, Chapel Hill, NC, USA, ${ }^{3}$ Department of Epidemiology, School of Public Health, University of North Carolina, Chapel Hill, NC, USA and ${ }^{4}$ Section of Preventive Medicine and Epidemiology, Boston University Medical Center, Boston, MA, USA

Email: Beth D Weatherley - beth.weatherley@duke.edu; Lloyd E Chambless - wchambless@mail.cscc.unc.edu; Gerardo Heiss* - gerardo_heiss@unc.edu; Diane J Catellier - diane_catellier@mail.cscc.unc.edu; Curtis R Ellison - ellison@bumc.bu.edu

* Corresponding author
\end{abstract}

Published: 2I February 2006

BMC Cardiovascular Disorders2006, 6:7 doi:10.1 |86/147|-226I-6-7

This article is available from: http://www.biomedcentral.com/|47I-226I/6/7

(c) 2006Weatherley et al; licensee BioMed Central Ltd.

This is an Open Access article distributed under the terms of the Creative Commons Attribution License (http://creativecommons.org/licenses/by/2.0), which permits unrestricted use, distribution, and reproduction in any medium, provided the original work is properly cited.
Received: 24 May 2005

Accepted: 21 February 2006

\begin{abstract}
Background: $A$ low ankle-brachial index $(A B I)$ is associated with increased risk of coronary heart disease, stroke, and death. Regression model parameter estimates may be biased due to measurement error when the $A B I$ is included as a predictor in regression models, but may be corrected if the reliability coefficient, $R$, is known. The $R$ for the ABI computed from DINAMAPTM readings of the ankle and brachial SBP is not known.
\end{abstract}

Methods: A total of II 9 participants in both the Atherosclerosis Risk in Communities (ARIC) study and the NHLBI Family Heart Study (FHS) had repeat ABls taken within I year, using a common protocol, automated oscillometric blood pressure measurement devices, and technician pool.

Results: The estimated reliability coefficient for the ankle systolic blood pressure (SBP) was 0.68 (95\% Cl: $0.57,0.77)$ and for the brachial SBP was $0.74(95 \% \mathrm{Cl}: 0.62,0.83)$. The reliability for the $A B I$ based on single ankle and arm SBPs was $0.6 \mathrm{I}(95 \% \mathrm{Cl}: 0.50,0.70)$ and the reliability of the ABI computed as the ratio of the average of two ankle SBPs to two arm SBPs was estimated from simulated data as 0.70 .

Conclusion: These reliability estimates may be used to obtain unbiased parameter estimates if the $A B I$ is included in regression models. Our results suggest the need for repeated measures of the $A B I$ in clinical practice, preferably within visits and also over time, before diagnosing peripheral artery disease and before making therapeutic decisions.

\section{Background}

The ratio of the ankle to the brachial systolic blood pressure (SBP), the ankle-brachial index (ABI), is an indicator of atherosclerotic vascular disease in the lower extremities and a simple, non-invasive measure of subclinical atherosclerosis [1-9]. The ABI has been shown in cross-sectional studies to be associated with cardiovascular disease (CVD) risk factors including smoking [10-17] diabetes [10- 
$12,18,19]$, total cholesterol $[10,12,13,20,21]$ hypertension $[10,12,13]$ and low birth weight $[14]$. The ABI is associated with other subclinical [10,22-25] and clinical $[10,12,13,23,25-28]$ CVD manifestations. Prospective studies have found that those with ABI-defined lower extremity arterial disease (LEAD) are approximately 1.5 to 2 times more likely to have a clinical CVD event $[27,29$ $31]$. The ABI has a graded, inverse association with mortality $[32,33]$.

Regression parameters may be biased when predictors are measured with error [34,35]. The parameter estimate for a regression model with a single explanatory variable measured with error will be biased towards the null by a multiplicative factor $R$, the reliability coefficient. Parameter estimates for additional covariates included in the model may be biased in any direction. Unbiased parameter estimates may be obtained using Stein estimators of true values [36] or other techniques if $R$ is known [34,35].

To measure ankle SBP, Doppler ultrasound devices have been used for many years to detect blood flow distal to an occluding cuff. Many epidemiologic CVD studies have included an ABI measured using either Doppler at the ankle and sphygmomanometry at the arm, or Doppler at both the ankle and the arm. In order to reduce interobserver variation, several studies including the Atherosclerosis Risk in Communities (ARIC) study [37] and the NHLBI Family Heart Study (FHS) [38] have employed an automated, oscillometric device to measure ankle and brachial SBPs. Limited data regarding the repeatability of ankle and arm SBPs measured with oscillometric devices are available, but the repeatability of the ABI measured with the widely-used DINAMAP ${ }^{\mathrm{TM}}$ has not been published to our knowledge.

DINAMAP ${ }^{\mathrm{TM}}$ ankle and brachial SBPs were taken during ultrasound exams using the same protocol, equipment, and pool of sonographers in the ARIC study and the FHS. Using repeat measures of the ABI taken within a year apart for each of these studies, we estimate variance components and reliability coefficients for ankle and brachial SBPs and for the ABI. We also examined the effect of participant characteristics on the ABI reliability.

\section{Methods}

\section{Study population}

The ARIC study cohort comprises 15,792 members aged 45-64 years of randomly selected households in four United States communities: Forsyth County, North Carolina; northwest suburbs of Minneapolis, Minnesota; Washington County, Maryland; and African American residents of Jackson, Mississippi [37,39]. ARIC participants were examined at baseline between December 1986 and January 1990 and were then examined every 3 years after their baseline examination. Institutional review board (IRB) approval for each examination cycle and for annual follow-up were obtained by each participating field center and the coordinating center. Informed consent itemized the procedures consented to and any restrictions to the use of biospecimen. The FHS population comprises 588 randomly-selected individuals (probands) and their families and 657 families with high coronary heart disease (CHD) risk scores from three ongoing epidemiologic studies in four communities: the Forsyth County, North Carolina and Minneapolis, Minnesota cohorts of the ARIC study; the Framingham Heart Study in Framingham, Massachusetts; and the Health Family Tree Program in Salt Lake City, Utah [38]. FHS participants were examined in 1994-1995. IRB approval for the examination and a follow-up were obtained by the participating field centers and the coordinating center. Informed consent itemized the procedures consented to and as well as restrictions to the use of biospecimens.

Seven hundred ten ARIC study participants and their families participated in the FHS clinic examination: 267 randomly-selected families, 340 families with high family risk scores, and 103 black participants at Forsyth [38]. One member of each selected ARIC household was designated as proband; 585 total ARIC probands were examined in Phase II of the FHS, of whom 577 had an ARIC 6or 9-year follow-up visit when the ABI was to be measured.

Replicate ankle-brachial index measures taken for each of the two studies were available for 335 ARIC probands, 120 of which were taken within 365 days. The ARIC ABI measure closest to the FHS exam was chosen; 48 participants had 3 measures, but in all cases the second and third measures were $>365$ days apart. One participant who had a difference between ABI measures that was more than 3 standard deviations (SDs) from the mean pair difference was excluded. Of the remaining 119 participants, 25 (21 percent) had their first measure in the FHS and the second at ARIC visit 3 and 22 (18 percent) had the second at ARIC visit $4 ; 72$ (61 percent) had the first ABI measure at ARIC visit 3 and the second at the FHS exam. Measures were taken an average of 228 days apart.

\section{Ankle-brachial index measurement}

Ankle and brachial blood pressures were obtained immediately prior to the ultrasound examinations by the same pool of 11 sonographers in both the FHS and ARIC follow-up visit clinic exams, using the same protocol and equipment $[40,41]$. With the participant resting in the supine position, the cuff was placed using a contour wrapping technique over the posterior tibial artery of one ankle [42], selected based on the date. The cuff was then placed over the brachial artery of the right arm, if anatomy per- 
Table I: Characteristics of ABI reliability study subjects compared with the ARIC study cohort

\begin{tabular}{|c|c|c|c|c|}
\hline \multirow[b]{2}{*}{ Characteristic } & \multicolumn{2}{|c|}{ ARIC baseline survey } & \multicolumn{2}{|c|}{ ARIC visit 3} \\
\hline & $\begin{array}{l}\text { ARIC Minneapolis and } \\
\text { Forsyth County } \\
(\mathbf{N}=\mathbf{8 0 4 4})\end{array}$ & $\begin{array}{c}\text { ABI reliability study } \\
(\mathbf{N}=119)\end{array}$ & $\begin{array}{l}\text { ARIC Minneapolis and } \\
\text { Forsyth County } \\
(\mathbf{N}=6839)^{\dagger}\end{array}$ & $\begin{array}{c}\text { ABI reliability study } \\
(\mathrm{N}=119)\end{array}$ \\
\hline Age, $y^{*}$ & $54.2(5.79)$ & $55.0(5.68)$ & $60.2(5.73)$ & $61.0(5.67)$ \\
\hline Female, \% & 52.8 & 58.8 & 53.4 & 58.8 \\
\hline \multicolumn{5}{|l|}{ Race/ethnicity, \% } \\
\hline White & 93.3 & $84.0 \S$ & 94.5 & $84.0 \S$ \\
\hline African American & 6.3 & 16.0 & 5.1 & 16.0 \\
\hline American or Alaskan Indian & 0.1 & 0 & 0.1 & 0 \\
\hline Asian or Pacific Islander & 0.3 & 0 & 0.3 & 0 \\
\hline \multicolumn{5}{|l|}{ Field center, \% } \\
\hline Forsyth County, NC & 50.2 & $64.7 \ddagger$ & 48.9 & $64.7 \S$ \\
\hline Minneapolis, Minn & 49.8 & 35.3 & 51.1 & 35.3 \\
\hline Weight, kg* & $76.0(16.21)$ & $75.7(15.63)$ & $78.1(16.79)$ & $78.4(17.12)$ \\
\hline Body mass index, $\mathrm{kg} / \mathrm{m}^{2 *}$ & $26.7(4.73)$ & $27.0(4.78)$ & $27.6(5.03)$ & $28.1(5.34)$ \\
\hline Diabetes, $\%$ & 6.7 & 7.6 & 12.0 & 11.8 \\
\hline \multicolumn{5}{|l|}{ Smoking status, \% } \\
\hline Current & 26.8 & 23.5 & 18.2 & 19.3 \\
\hline Former & 35.7 & 33.6 & 44.8 & 42.9 \\
\hline Never & 37.5 & 42.9 & 37.0 & 37.8 \\
\hline Hypertension, \% & 26.6 & 26.9 & 34.4 & 35.3 \\
\hline Coronary heart disease, \% & 4.6 & $18.0 \S$ & 6.8 & $23.7 \S$ \\
\hline Ankle brachial index* & $1.14(0.130)$ & $1.13(0.137)$ & $1.19(0.150)$ & $1.17(0.150)$ \\
\hline
\end{tabular}

*Numbers presented are mean (SD).

† ARIC participants with a visit 3 (6-year follow-up); for the ABI, N = 2295 .

$\ddagger 0.001 \leq p<0.01$ for chi-square test comparing sample to source study population.

$\S p<0.001$

mitted. Blood pressures were taken with a DINAMAP ${ }^{\mathrm{TM}}$ 1846 SX automated oscillometric device (Critikon, Inc., Tampa, FL). None of the 577 subjects in both studies had a recorded blood pressure outside the device's detection limits, $<30$ or $>245 \mathrm{mmHg}$ [43]; one subject was excluded who had one $\mathrm{ABI}$ where the ankle systolic blood pressure (SBP) was $\geq 75 \mathrm{mmHg}$ more than the brachial SBP, a criterion employed to exclude non-compressible arteries [44]. The ABI was computed as the ratio of the ankle SBP to the brachial SBP.
At the ARIC baseline survey, the ankle SBP was measured with the participant prone before and after ultrasound scanning of the popliteal artery, then brachial SBPs were measured supine every 5 minutes during the carotid ultrasound. The ABI was computed as the ratio of the average of the last two available ankle SBPs to the average of the first two brachial SBPs.

\section{Covariates}

In the ARIC study and the FHS, hypertension was defined as a sitting SBP > $140 \mathrm{mmHg}$, diastolic blood pressure >

Table 2: Variation in DINAMAP ${ }^{\mathrm{TM}}$ measurements of arm and ankle systolic blood pressure and the ankle-brachial index

\begin{tabular}{|c|c|c|c|}
\hline Estimate & Ankle SBP & Arm SBP & ABI \\
\hline Mean (SD) of pair means, $\mathrm{mmH}^{*}{ }^{*}$ & $146.2(23.76)$ & $125.2(19.57)$ & $1.178(0.1522)$ \\
\hline Mean (SD) of pair differences, $\mathrm{mmH} \mathrm{g}^{*}$ & $-3.1(20.67)$ & $0.9(15.24)$ & $-0.031(0.1494)^{\ddagger}$ \\
\hline \multicolumn{4}{|l|}{ Square root of variance components, $\mathrm{mmH} \mathrm{g}^{*}$} \\
\hline Between-person ${ }^{\dagger}$ & $21.4(17.6,25.1)$ & $18.0(14.5,1.3)$ & $0.133(0.112,0.150)$ \\
\hline Within-person ${ }^{\dagger}$ & $14.6(12.7,16.5)$ & $10.8(8.9,12.4)$ & $0.106(0.091,0.121)$ \\
\hline Total ${ }^{\dagger}$ & $25.9(22.8,28.7)$ & $21.0(18.3,3.7)$ & $0.170(0.151,0.185)$ \\
\hline Reliability coefficient ${ }^{\dagger}$ & $0.682(0.570,0.772)$ & $0.736(0.622,0.827)$ & $0.612(0.505,0.699)$ \\
\hline Within-person coefficient of variation, $\%^{\dagger}$ & $10.0(8.6,11.2)$ & $8.6(7.1,9.9)$ & $9.0(7.7,10.2)$ \\
\hline Pearson correlation coefficient & 0.68 & 0.74 & 0.62 \\
\hline
\end{tabular}

*Systolic blood pressures (SBPs) in mmHg; the ankle-brachial index (ABI) is without units.

Difference represents first minus second measure.

tEstimate ( $95 \%$ confidence interval).

$\ddagger p<.05$ for t-test of zero mean. Adjustment for the fixed effect of time changed very little the estimates of variance components. 
Table 3: Variation in DINAMAP ${ }^{\mathrm{TM}}$ measurements of the ankle-brachial index by subgroup defined at the first measurement

\begin{tabular}{|c|c|c|c|c|c|c|}
\hline \multirow[b]{2}{*}{ Subgroup } & \multirow[b]{2}{*}{$\mathbf{N}$} & \multicolumn{3}{|c|}{ Square root of variance components } & \multirow[b]{2}{*}{ Reliability Coefficient } & \multirow[b]{2}{*}{ Within-person CV, \% } \\
\hline & & Between-person & Within-person & Total & & \\
\hline Overall & 119 & 0.133 & 0.106 & 0.170 & 0.612 & 9.0 \\
\hline \multicolumn{7}{|l|}{ Age, $y$} \\
\hline$\leq 62$ & 66 & 0.124 & 0.104 & 0.162 & 0.587 & 8.9 \\
\hline$>62$ & 53 & 0.144 & 0.107 & 0.180 & 0.643 & 9.1 \\
\hline \multicolumn{7}{|l|}{ Race/ethnicity } \\
\hline African American ${ }^{\dagger}$ & 19 & 0.098 & 0.079 & 0.125 & 0.606 & 6.8 \\
\hline White & 100 & 0.139 & 0.110 & 0.177 & 0.614 & 9.3 \\
\hline \multicolumn{7}{|l|}{ Gender } \\
\hline Male & 49 & 0.140 & 0.102 & 0.173 & 0.653 & 8.4 \\
\hline Female & 70 & 0.122 & 0.109 & 0.163 & 0.555 & 9.5 \\
\hline \multicolumn{7}{|l|}{ Field center } \\
\hline Forsyth County, NC & 77 & 0.127 & $0.116 *$ & 0.172 & 0.545 & $9.9 *$ \\
\hline Minneapolis, Minn & 42 & 0.142 & $0.086 *$ & 0.166 & 0.732 & $7.2^{*}$ \\
\hline \multicolumn{7}{|l|}{ Body mass index, $\mathrm{kg} / \mathrm{m}^{2}$} \\
\hline$\leq 27$ & 61 & 0.141 & $0.083^{*}$ & 0.164 & $0.74 I^{*}$ & $7.1^{*}$ \\
\hline$>27$ & 58 & 0.124 & $0.126 *$ & 0.178 & $0.495^{*}$ & $10.7^{*}$ \\
\hline \multicolumn{7}{|l|}{ Diabetes } \\
\hline No & 106 & 0.134 & 0.099 & 0.167 & 0.646 & 8.4 \\
\hline Yest & 13 & 0.112 & 0.150 & 0.187 & 0.358 & 13.3 \\
\hline \multicolumn{7}{|l|}{ Hypertension } \\
\hline No & 76 & 0.128 & 0.100 & 0.163 & 0.622 & 8.3 \\
\hline Yes & 43 & 0.134 & 0.116 & 0.177 & 0.574 & 10.1 \\
\hline \multicolumn{7}{|l|}{ Hypertension rx switch $\ddagger$} \\
\hline No & 101 & 0.136 & 0.104 & 0.171 & 0.630 & 8.8 \\
\hline Yes $^{\dagger}$ & 18 & 0.114 & 0.115 & 0.162 & 0.498 & 9.9 \\
\hline \multicolumn{7}{|l|}{ Coronary heart disease } \\
\hline No & 88 & 0.129 & 0.098 & 0.162 & 0.638 & 8.3 \\
\hline Yes & 31 & 0.148 & 0.116 & 0.188 & 0.619 & 9.9 \\
\hline \multicolumn{7}{|l|}{ Legs } \\
\hline Same & 51 & 0.115 & 0.118 & 0.165 & $0.486^{*}$ & 10.0 \\
\hline Different & 68 & 0.146 & 0.094 & 0.174 & $0.706^{*}$ & 8.6 \\
\hline \multicolumn{7}{|l|}{ Days between measures } \\
\hline$\leq 240$ & 60 & 0.131 & 0.107 & 0.169 & 0.602 & 9.1 \\
\hline$>240$ & 59 & 0.136 & 0.103 & 0.171 & 0.633 & 8.7 \\
\hline \multicolumn{7}{|l|}{ FHS sampling group } \\
\hline African American $\dagger$ & 19 & 0.098 & 0.079 & 0.125 & 0.606 & 6.8 \\
\hline High family risk score & 68 & 0.148 & 0.114 & $0.187^{*}$ & 0.629 & 9.7 \\
\hline Random sample & 32 & 0.117 & 0.099 & $0.153^{*}$ & 0.581 & 8.2 \\
\hline
\end{tabular}

${ }^{*} p<0.05$ for the difference between the two subgroups (the $95 \%$ bootstrapped confidence interval for the difference did not include zero).

tProportion too small to perform statistical tests of differences.

\#"No" includes those who were either taking or not taking hypertension medication at the time of both ABI measures; "yes" includes those who were taking hypertension medication at the time of one $A B I$ measure and not taking hypertension medication at the time of the other $A B I$ measure. 


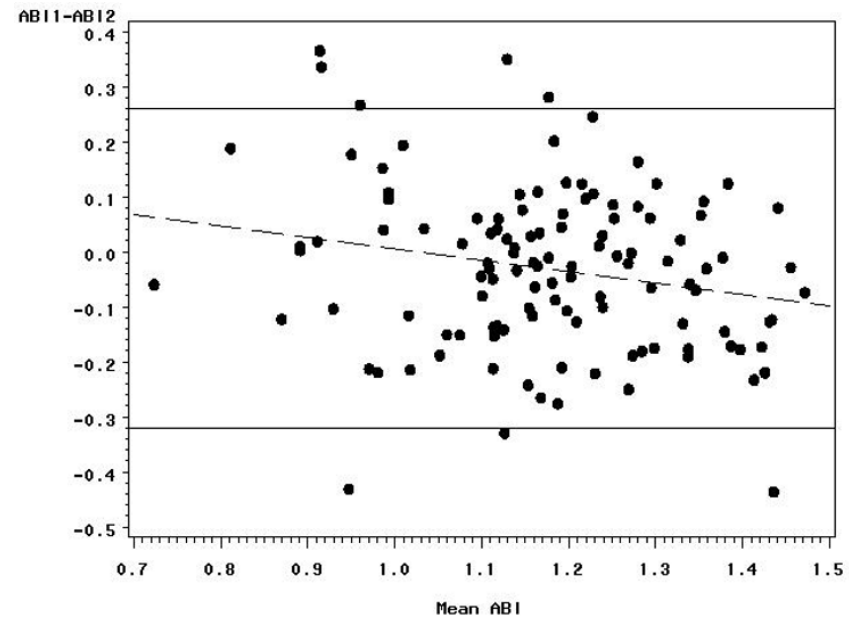

Figure I

Bland-Altman plot showing the reproducibility of the ankle-brachial index. The differences between the first and second measurements are plotted against the mean of the two measures with $95 \%$ limits of agreement (solid lines) and regression line (dashed line).

$90 \mathrm{mmHg}$, or use of antihypertensive medication within 2 weeks prior to the examination. Diabetes was defined as a fasting glucose level $\geq 7.8 \mathrm{mmol} / \mathrm{L}(140 \mathrm{mg} / \mathrm{dL})$, a nonfasting level $\geq 11.1 \mathrm{mmol} / \mathrm{L}(200 \mathrm{mg} / \mathrm{dL})$, self-reported history of diabetes, or the use of hypoglycemic agents. In ARIC, prevalent CHD was defined as evidence of a prior myocardial infarction on a 12-lead ECG, or self-reported history of a physician-diagnosed heart attack, coronary bypass surgery, or coronary angioplasty. In the FHS selfreported, physician diagnosed coronary heart disease was confirmed by review of hospital records.

\section{Statistical analysis}

For all statistical tests, two-sided $p$ values $<0.05$ were considered statistically significant. Characteristics of the ABI reliability study subjects were compared to characteristics of the ARIC source population at visits 1 and 3 using Kruskal-Wallis two-sample tests for continuous variables and Wilcoxon tests for categorical variables.

Each of the two observed values was assumed to represent the sum of the true value and random within-person variation [34]. Within-person variation may be due to measurement error and physiologic variation. We assumed that the within-person variation is independent of the value's magnitude and that the within-person variation for each of the two values are independent and identically distributed with mean zero and common variance. Estimates of the between-person and within-person variances were obtained with estimators derived using the method of moments; similar estimates were obtained using SAS
PROC MIXED release 8.1 (SAS Institute, Inc., Cary, NC). The reliability coefficient, $R$, was computed as the ratio of the between-person variance to the total variance and can be interpreted as the proportion of the total variance not due to within-person variation, or as the correlation between measures made at repeat visits for an individual. The within-person coefficient of variation $(\mathrm{CV})$ was computed as 100 times the square root of the within-person variance divided by the mean of the individual mean replicates. These methods were applied to ankle and brachial SBP measurements as well as to ABI. In addition, the multivariate generalization of this method was applied to produce the multivariate error covariance matrices and between-person covariance matrices for ankle and arm SBP and the component-wise ratios of the error and between-person covariance to total covariance. (The diagonal elements of this matrix of ratios are the reliability coefficients for ankle and arm SBP).

Because the arm SBPs and ABIs were not normally distributed, confidence intervals for the estimates of variance components and reliability coefficients were derived from 300 bootstrap samples [45]. We compared variance components, reliability coefficients, and CVs by levels of important covariates, including the FHS sampling group. Analyses did not account for the FHS sampling scheme. To test subgroup differences, we constructed bootstrapped 95 percent confidence intervals for the differences; intervals not including zero were deemed statistically significant.

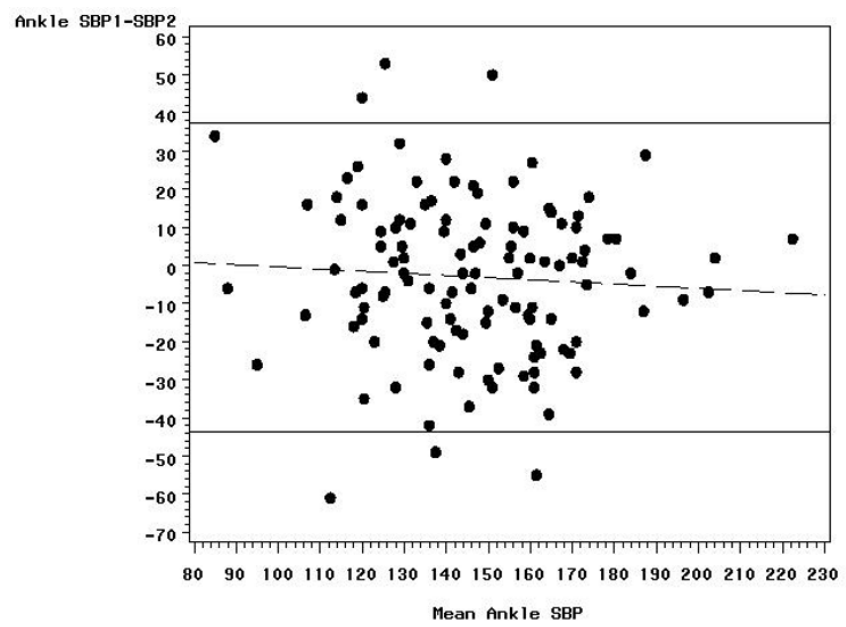

\section{Figure 2}

Bland-Altman plot showing the reproducibility of the ankle systolic blood pressure. The differences between the first and second measurements are plotted against the mean of the two measures with $95 \%$ limits of agreement (solid lines) and regression line (dashed line). 
To examine the assumption of independence of the within-person variation from the ABI level, we compared the estimated square root of the within-person variance (i.e., the within-person SD) for those whose first of the two repeat ABIs was at or below with those whose first ABI was above the median $\mathrm{ABI}$ in the 119 reliability study subjects. We also examined plots of the pair differences versus the pair means for the ankle SBP, arm SBP, and ABI [46].

The ABI measured at the ARIC baseline survey is of interest as a predictor of subsequent events. Because repeat ankle and arm SBPs are available for the baseline survey, the $\mathrm{ABI}$ is usually computed as the ratio of the average of two ankle SBPs (in the same ankle) to the average of two arm SBPs (in the right arm). We estimated the within-visit multivariate error and total covariance matrices using repeat ankle and arm SBP measures taken at the ARIC baseline survey. The ratios of within-visit multivariate error to total covariance matrices from the baseline survey data were multiplied times the total variance in the ARIC/ FHS repeat data to partition the non-between person variance found there into between-visit and within-visit components. We simulated a two-measures-per-visit repeatability dataset very much like the actual repeatability dataset by taking each subject's mean of the two actual repeated BP measures as the "true" BP values, and adding independent random "between-visit" and "within-visit" Gaussian measurement error, of size to make these two variance components the same proportion of the total variance as in the results of the repeatability study and the baseline survey within-visit repeatability analysis. With the simulated data, the reliability coefficient for the $\mathrm{ABI}$ was computed as a ratio of means, along with the reliability coefficient using just the first measure at each visit. This was repeated 1000 times and the reliability coefficients averaged.

\section{Results}

Of the 119 reliability study subjects, 70 (58.8 percent) were women, 100 (84.0 percent) were white, and 77 (64.7 percent) were from the Forsyth County field center. At the time of the first $A B I$ measure, the mean age was 61.3 years, mean weight $75.7 \mathrm{~kg}$, mean body mass index $28.0 \mathrm{~kg} / \mathrm{m}^{2}$, and mean ABI 1.16; 13 (10.9 percent) had diabetes mellitus, 43 (36.1 percent) had hypertension, and 31 (26.0 percent) had coronary heart disease. Characteristics of this group were similar to those of the ARIC cohort in Minneapolis and Forsyth County at the time of the ARIC baseline survey and at visit 3 , except with respect to the distribution of characteristics related to the FHS sampling procedures: race/ethnicity, field center, and CHD prevalence (Table 1).

The estimated reliability coefficient for the ankle SBP was 0.682 (95 percent confidence interval (CI): 0.570, 0.772),

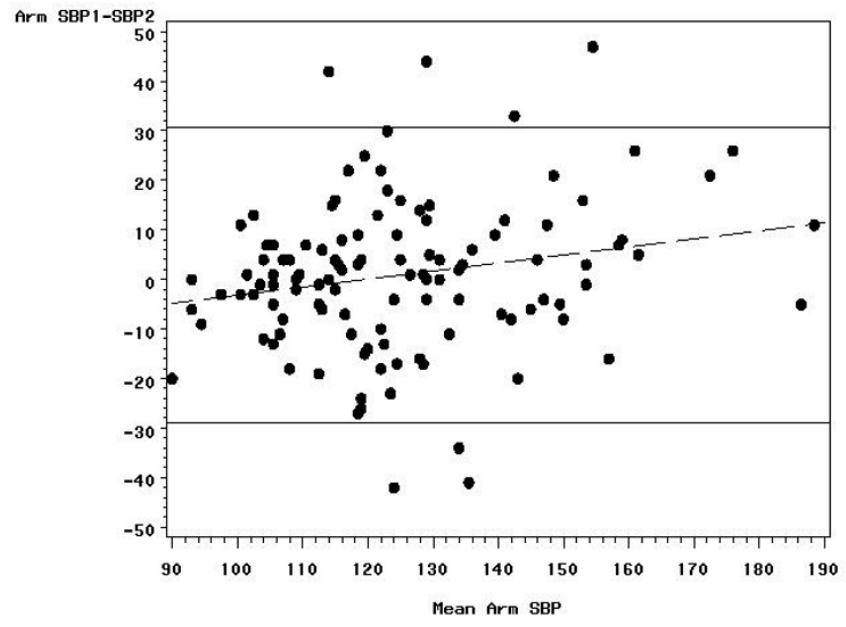

\section{Figure 3}

Bland-Altman plot showing the reproducibility of the brachial systolic blood pressure. The differences between the first and second measurements are plotted against the mean of the two measures with $95 \%$ limits of agreement (solid lines) and regression line (dashed line).

for the arm SBP was 0.736 (95 percent CI: 0.622, 0.827), and for the ABI was 0.612 (95 percent CI: 0.505, 0.699) (Table 2). These values are very close to estimated Pearson correlation coefficients of $0.68,0.74$, and 0.62 , respectively, which have no underlying measurement error model assumptions. Arm SBP measures appear to have been more repeatable than ankle SBPs, as reflected by a lower within-person SD (10.8 v. $14.6 \mathrm{mmHg})$, a lower within-person CV (8.6 v. 10.0 percent), and a higher reliability coefficient (0.736 v. 0.682). Exclusion of 4 data pairs with either ABI measurement $>1.5$ had little effect on variance estimates.

The ankle SBP increased an average of $3.1 \mathrm{mmHg}$ between the first and second measures $(p=0.11)$, which resulted in a statistically significant average increase of 0.031 in the $\operatorname{ABI}(p=0.02)$. Adjustment for the fixed effect of time (first v. second measure) in an analysis of variance model changed the variance component estimates very little. FHS ankle SBP measures were an average of $3.2 \mathrm{mmHg}$ higher than ARIC measures $(p=0.10)$. ABI measures in the FHS were 0.029 higher, on average, than ARIC measures $(p=$ $0.03)$; controlling for the fixed effect of time reduced the study difference to $0.024(p=0.09)$. No significant effects of time or study were apparent for arm SBP.

The estimated ABI variance components differed in some subgroups (Table 3). The within-person SD was statistically significantly smaller at the Minneapolis than at the Forsyth County field center (0.086 versus 0.116$)$. The within-person SD was smaller in those with a $\mathrm{BMI} \leq$ the 
Table 4: Multivariate between-visit covariance matrices for ankle and arm systolic blood pressures estimated in reliability study subjects

\begin{tabular}{lcc}
\hline & Ankle SBP & Arm SBP \\
\hline Within-person covariance & & \\
$\quad$ Ankle SBP & 213.6 & 88.3 \\
$\quad$ Arm SBP & 88.3 & 116.2 \\
& & \\
True/total ratio matrix & & 0.741 \\
$\quad$ Ankle SBP & 0.682 & 0.736 \\
Arm SBP & 0.741 & \\
\hline
\end{tabular}

median of $27 \mathrm{~kg} / \mathrm{m}^{2}$ than in those with a BMI > $27 \mathrm{~kg} / \mathrm{m}^{2}$ $(0.083$ versus 0.126$)$, resulting in a significantly greater reliability coefficient in those with a lower compared to those with a higher BMI (0.741 versus 0.495$)$. Those whose same ankles were measured had a statistically significantly lower reliability coefficient $(R=0.486)$ than those with different ankles measured $(R=0.706)$. There were too few African American subjects, subjects with diabetes, and subjects who initiated or discontinued hypertension treatment to test for subgroup differences. Withinperson SDs and reliability coefficients did not differ significantly by age $\leq$ or $>62$ years, gender, hypertension, or by whether the time between measures was greater or less than the median interval of 240 days. Except for the estimated total variance, estimates did not differ significantly between those in the high family risk score and the random sample FHS sampling groups; the proportion of reliability study subjects in the African American sampling group was too small to test for differences.

Within-person SD estimates did not differ significantly between those with an $\mathrm{ABI} \leq 1.15$ and those with an $\mathrm{ABI}$ $>1.15$ (0.113 versus 0.097), the median $\mathrm{ABI}$ in the 119 reliability study subjects. A plot of the pair differences versus the pair means revealed no obvious dependence of the spread of the differences on the ABI level (Figures 1, 2, 3); however, the difference between the first and second measure decreased with increasing ABI level. The ankle SBP differences did not depend statistically significantly on ankle SBP level, but the arm pressure difference decreased with increasing arm SBP. The two ankle pressures differed by $>10 \mathrm{mmHg}$ in 73 (61.3 percent) subjects, by $>20 \mathrm{mmHg}$ in 38 (31.9 percent) subjects, by $>30$ mmHg in 15 (12.6 percent) subjects, and by $>40 \mathrm{mmHg}$ in 7 (5.9 percent) subjects. Arm pressures differed by $>10$ $\mathrm{mmHg}$ in 51 (42.9 percent) subjects, by $>20 \mathrm{mmHg}$ in 19 (16.0 percent) subjects, by $>30 \mathrm{mmHg}$ in 7 (5.9 percent) subjects, and by $>40 \mathrm{mmHg}$ in 5 (4.2 percent) subjects.

The multivariate within-person error covariance and trueto-total ratio covariance matrices for ankle and arm SBPs estimated from the reliability study are given in Table 4. The non-zero ankle-arm covariance suggests that the ankle and arm SBP within-person errors were correlated $(\rho=$ 0.56). The diagonal elements of the true-to-total component-wise ratio covariance matrix represent the scalar reliability coefficients.

The multivariate within-person (between-visit) error covariance and true-to-total component-wise ratio covariance matrices estimated from the ARIC baseline survey are given in Table 5. The within-visit ankle and arm errors were essentially uncorrelated $(\rho=0)$. The correlation between within-visit measures was high: 0.92 for ankle SBP and 0.90 for arm SBP.

From ARIC baseline survey data, we also estimated the within-visit variance and reliability of repeat ABIs, arbitrarily computed as the ratio of the first ankle to first arm SBP and the ratio of the second ankle to second arm SBP. The within-visit error variance for the ABI was 0.00636, and the reliability coefficient was 0.681 .

The ABI measured at the ARIC baseline survey, computed as the ratio of the average of two ankle SBPs to the average of two arm SBPs, is of interest as a predictor in regression models. Using the estimates of the total and within-visit errors (Tables 4 and 5, respectively), a dataset containing two ankle and two arm SBPs at each of two visits was simulated by assuming that the pair means for the reliability study represented the "true" values. For each of 1000 replications, the reliability coefficients were estimated for the $\mathrm{ABI}$ computed at each visit as the ratio of the averages of the within-visit values and the ABI computed as the ratio of the first measures, and then the mean reliability coefficients over the replications taken. The result for the reliability coefficient for single measures was 0.613 , very near that from the actual reliability study, and was 0.704 for the reliability coefficient for the ratio of means.

\section{Discussion}

The within-person variance estimated from our data indicates that the $95 \%$ confidence interval of a patient's ABI would be the $A B I$ value \pm 0.21 if based on a single measure of the ABI. Thus, our results suggest the need for repeated measures of the $\mathrm{ABI}$ in clinical practice, if at all possible 
Table 5: Multivariate within-visit covariance matrices for ankle and arm systolic blood pressures estimated in ARIC study participants

\begin{tabular}{lcc}
\hline & Ankle SBP & Arm SBP \\
\hline Within-person covariance & & -0.0159 \\
$\quad$ Ankle SBP & 49.8 & 44.0 \\
$\quad$ Arm SBP & -0.0159 & \\
& & 1.000 \\
True/total ratio matrix & & 0.900 \\
$\quad$ Ankle SBP & 0.922 & 1.000 \\
$\quad$ Arm SBP & & \\
\hline
\end{tabular}

within visits and also over time, before diagnosing peripheral artery disease and before making therapeutic decisions.

To our knowledge, few studies of the repeatability of ankle or arm pressures measured with the DINAMAP ${ }^{\mathrm{TM}}$ have been published, and the designs of the few published studies do not include these same sources of variability. No reliability coefficient has been published, of which we are aware, for the $\mathrm{ABI}$ measured using the DINAMAP ${ }^{\mathrm{TM}}$ at both the ankle and arm.

We have estimated within-person SDs of $14.6 \mathrm{mmHg}$ for ankle SBP and $10.8 \mathrm{mmHg}$ for brachial SBP, with reliability coefficients of 0.68 and 0.74 , respectively, for DINAMAP ${ }^{\mathrm{TM}}$ measures taken up to 1 year apart in a sample of two middle-aged populations. Using a DINAMAP ${ }^{\text {тм }}$ 1846SX and a contour wrap at the ankle, Mundt et al [42] found a within-person SD of $4.0 \mathrm{mmHg}$ for ankle SBP and $2.5 \mathrm{mmHg}$ for brachial SBP, with reliability coefficients of 0.94 and 0.92 , respectively, for three repeat ankle and two repeat arm measures one minute apart by one of two technicians among 71 adult volunteers aged 23-67 years; the same ankle was used for all repeat measures. Ramanathan et al [47] who also used a contour wrap at the ankle but used a VitalCare DOX Model 506DXN automated oscillometric device, estimated inter-reading intraclass correlation coefficients (ICCs) of 0.83 for ankle SBP and 0.85 for arm SBP for 3 repeat measures taken 30 minutes apart by each of 2 investigators among 50 healthy volunteers with a median age of 23 years. De Graaff et al [48] using a DINAMAP ${ }^{\mathrm{TM}}$ Plus measured brachial SBPs twice by each of two observers at each of 2 visits one week apart in 54 vascular laboratory patients with a mean age of 66 years. Estimates of within- and between-day, and within- and between-week, ICCs are given, but no overall estimate is presented with which we can compare our estimate of reliability.

Using the contour wrapping technique, ankle SBP measured with the DINAMAP ${ }^{\mathrm{Tm}} 1846$ SX were found to be about $3.0 \mathrm{mmHg}$ lower, on average, than measures taken with Doppler ultrasound [42]. Estimates of reliability were comparable between the two methods, with a reliability coefficient of 0.92 for Doppler and 0.94 for DINAMAP ${ }^{\mathrm{rm}}$ measures. This study did not include variation due to different days or legs, however. Fowkes et al [49] studied the variability of Doppler ultrasound measures of ankle SBP, brachial SBP, and the ABI in 24 peripheral artery disease (PAD) patients and 11 volunteers without PAD aged 4074 years. Two measures were taken in each leg and the right arm by each of two observers and then repeated two weeks later. Reliability coefficients can be computed from the results by summing all intraindividual variance components. Regrettably, components of variance $<10$ were not reported, but the range of possible values can be estimated assuming unreported estimates were either all 10 or all 0. For Doppler ankle SBP, $R$ was between 0.78 and 0.90 for normal subjects, and 0.37 and 0.46 for diseased subjects. For Doppler arm SBP, $R$ was between 0.87 and 0.91 for normal subjects, and was 0.68 for diseased subjects. For the ABI, $R$ was between 0.40 to 0.42 for diseased subjects; the range of $R$ computed from the reported between-subject variance for normal subjects was implausibly low. These values bracket those found in our reliability study for DINAMAP ${ }^{\mathrm{TM}}$ SBP measures.

The estimated reliability coefficient for the ABI, 0.61, is comparable to that for another measure of subclinical atherosclerosis in the ARIC study, carotid intima-medial thickness (IMT), but is lower than that found for other measures. A reliability coefficient of 0.60 was estimated for the mean carotid IMT, averaged over 3 arterial sites bilaterally, in 279 subjects who participated in both the ARIC study and the FHS and with carotid B-mode ultrasounds within a year apart (Diane Catellier, University of North Carolina, unpublished manuscript, 2000). A reliability coefficient of 0.67 was estimated for the mean carotid IMT measured at three visits, 7-14 days apart in 36 volunteers from each of the four ARIC field centers [50]; measures of arterial distensibility and arterial compliance had reliability coefficients of 0.67 and 0.77 [51]. Estimated reliability coefficients for the ARIC study are 0.94 for both total and HDL-cholesterol [52], and 0.72 for plasma fibrinogen [53].

The within-person variation in this reliability study includes variation due to within and between observer, 
day, and leg, and physiologic variation. Thus, the ABI reliability estimated from the reliability study data is lower than the within-visit reliability estimated from ARIC baseline survey data, because the reliability study's within-person variability includes both between-visit and withinvisit variation. The between-visit variation includes variation due to different technicians, different machines, and within-person biologic variation.

The reliability of the ABI was lower than that of either of its components: the ankle SBP or arm SBP. The lower reliability of the ankle SBP compared with that of the arm SBP may be due to the greater difficulty in placing a cuff on the more conical extremity.

Estimates of within-person variation in this reliability study apply to measures using the methodology at ARIC visits 3 and 4 and in the FHS, and may not apply to other situations. Extreme care was taken in these studies to minimize measurement variation, including a detailed, common protocol and training and certification of technicians. Reliability is likely to be lower in patient care settings, where these methods are unlikely to be standardized. Different oscillometric devices may vary in the algorithms employed for determining systolic blood pressure [54] and the accuracy of different oscillometric devices relative to sphygmomanometry has been found to differ [55]; the repeatability of different devices may differ as well. In addition, the reliability coefficient depends on the total variance in the population and, therefore, the reliability estimated in this study may not apply to other populations. Assuming the same within-person variance, if the total variance is lower than in the reliability study, as it is in the ARIC baseline survey, then the reliability coefficient would be lower than that estimated.

The reliability study defined the ABI as a ratio of single blood pressure measurements. In the ARIC baseline survey, the ABI is defined using the mean of two ankle SBPs (in the same leg) and the mean of two arm SBPs (in the same arm), where the measures were taken approximately 5 minutes apart, and in the study of associations between the $\mathrm{ABI}$ and potential disease outcomes this $\mathrm{ABI}$ will be used. We have seen that the reliability coefficient for such an $\mathrm{ABI}$ is notably higher than that computed directly from the ARIC/FHS reliability study.

In clinical practice, repeated measures of the ABI should be made, preferably within visits and over time before diagnosing peripheral artery disease and before making therapeutic decisions. Based on the within-person variance estimated here, the 95\% confidence interval (1.96 times the within-person standard deviation) for one person based on one measure of the $\mathrm{ABI}$ would be the $\mathrm{ABI} \pm$ 0.21 . In general, for the average of $n$ measures, this inter- val becomes the ABI average $\pm 0.21 / \sqrt{ } n$, so the interval for an $\mathrm{ABI}$ based on the average of two visits would be $\mathrm{ABI} \pm$ 0.15 , and average $\mathrm{ABI} \pm 0.12$ if based on 3 measures. The reliability of oscillometric measurement of the brachial SBP is not much greater than that of the ABI, and multiple measures of the brachial SBP are recommended before instituting hypertension treatment [56].

Most population-based epidemiologic studies have measured the ankle pressure in both legs, once [18] or twice over the posterior tibial $[10,12,57]$ or both over this artery and the dorsalis pedis [58] on each ankle. The mean of the repeated measurements on each ankle $[10,12,57]$ or the highest SBP for each ankle [58] was then used, and a single $\mathrm{ABI}$ for the participant was computed using the minimum of the ankle numerators of the two legs. ABI values based on different protocols likely are associated with different levels of variability, the most robust (although not necessarily the most sensitive to peripheral arterial disease), being those based on averaged repeated measures. The effect of the choice of measurement protocol on the $\mathrm{ABI}$ reliability would be important to know, but cannot be assessed in this study since the ABI was defined in ARIC using ankle pressure measured in one, randomly selected leg.

The limited number of reliability study subjects in certain subgroups limited our ability to test for subgroup differences. The significantly higher within-person SD at Forsyth might be because 7 technicians were employed at the Forsyth County field Center and 4 at Minneapolis. The Forsyth County field center had 25 percent African American subjects, while Minneapolis subjects were all white ( $p$ $<0.001$ ); Forsyth also had a higher proportion of subjects with diabetes (14 percent v. 5 percent) than Minneapolis. The center difference might also reflect different proportions of subjects with higher within-person variation, although the difference in reliability coefficients between the two centers was not larger than chance alone would explain and small numbers in these subgroups prevent further analysis. Counter to intuition, the ABI had a lower reliability in those whose $A B I$ was measured in the same leg than in those whose was measured in different legs; this may represent a chance finding in this small study. It would have been desirable to examine whether the reliability differed in subjects with and without ABI-defined LEAD (e.g., $\mathrm{ABI}<0.9$ ), but the limited sample size did not allow for this analysis.

Individuals are often categorized as having LEAD or not by categorizing the observed $\mathrm{ABI}$ as below or above a cutpoint, typically 0.9. This categorical variable is then included as a predictor in regression models. An upper limit of normal for the ABI also has been proposed [57] to assess arterial stiffness and medial arterial calcification. 
Categorization of a continuous variable measured with non-differential measurement error may result in differential misclassification when the probability of disease is related to the level of the continuous variable [59]. Resulting regression parameter estimates are biased, but methods that assume non-differential misclassification may not correctly adjust these estimates.

\section{Conclusion}

The reliability of the ABI computed as the ratio of single ankle and arm oscillometric SBPs was found to be 0.61 . The reliability of the $\mathrm{ABI}$ computed as the ratio of the average of two ankle SBPs to two arm SBPs was estimated from simulated data as 0.70 . These reliability estimates may be used to obtain unbiased parameter estimates if the ABI is included in regression models. If ankle and arm SBP are included as separate predictors in a regression model, corrected parameter estimates may be obtained by taking the multivariate reliability into account.

\section{Competing interests}

The author(s) declare that they have no competing interests.

\section{Authors' contributions}

GH and BW conceived of and designed the study. BW and LC performed the statistical analyses. GH, LC, and BW interpreted the results. BW drafted the manuscript. All authors revised the manuscript for intellectual content, and read and approved the final manuscript.

\section{Acknowledgements}

The ARIC study is supported by National Heart, Lung, and Blood Institute contracts NOI-HC-550I5, NOI-HC-550I6, NOI-HC-550I8, NOI-HC55019 , NOI-HC-55020, NOI-HC-5502I, and NOI-HC-55022. A list of principal ARIC study staff was published in Am J Epidemiol (1989; 129:687688). The FHS is supported by National Heart, Lung, and Blood Institute contracts NOI-HC-25I04, NOI-HC-25I05, NOI-HC-25 I06, NOI-HC25I07, NOI-HC-25I08, and NOI-HC-25109. A list of principal FHS staff was published in Am J Epidemiol (1996; I 14: 1 227). This study was supported in part by an institutional National Research Service Award, contract 5T32-HL-07055 (Dr Weatherley).

\section{References}

I. Winsor T: Influence of arterial disease on the systolic blood pressure gradients of the extremity. Am J Med Sci 1950, 220:117.

2. Strandness DE, Bell JW: Peripheral vascular disease: diagnosis and objective evaluation using a mercury strain guage. Annals of Surgery 1965, 161 Suppl.:3.

3. Carter SA: Indirect systolic pressures and pulse waves in arterial occlusive disease of the lower extremities. Circulation 1968, 37:624.

4. Yao ST, Hobbs JT, Irvine WT: Ankle systolic pressure measurements in arterial disease affecting the lower extremities. British Journal of Surgery 1969, 56:676.

5. Carter SA: Clinical measurement of systolic pressure in limbs with arterial occlusive disease. JAMA 1969, 207:1869.

6. Fronek A, Johnson KH, Dilley RB, Bernstein EF: Non-invasive physiologic tests in the diagnosis and characterization of periph- eral arterial occlusive disease. American Journal of Surgery 1973, 126:205.

7. Ouriel K, Zarins CK: Dopper ankle pressure: an evaluation of three methods of expression. Arch Surg 1982, II 7: 1297.

8. Kiekara O, Riekkinen H, Soimakallio S, Lansimies E: Correlation of angiographically determined reduction of vascular lumen with lower-limb systolic pressures. Acta Chir Scand 1985, I I I:437.

9. Lijmer JG, Hunink MG, van den Dungen JJ, Loonstra J, Smit AJ: ROC analysis of noninvasive tests for peripheral arterial disease. Ultrasound in Medicine \& Biology 1996, 22(4):391.

10. Newman AB, Siscovick DS, Manolio TA, Polak J, Fried LP, Borhani NO, Wolfson SK: Ankle-arm index as a marker of atherosclerosis in the Cardiovascular Health Study. Circulation 1993, 88:837.

II. Beks PJ, Mackaay AJC, deNeeling JND, de Vries H, Bouter LM, Heine $\mathrm{RJ}$ : Peripheral arterial disease in relation to glycaemic level in an elderly Caucasian population: the Hoorn Study. Diabetologia 1995, 38:86.

12. Curb JD, Masaki K, Rodriguez BL, Abbott RD, Burchfiel CM, Chen R, Petrovitch H, Sharp D, Yano K: Peripheral artery disease and cardiovascular risk factors in the elderly. Arteriosclero Thromb Vasc Biol 1996, 16:1495-500.

13. Stoffers HEJH, Rinkens PE, Kester AD, Kaiser V, Knottnerus JA: The prevalence of asymptomatic and unrecognized peripheral arterial occlusive disease. International Journal of Epidemiology 1996, 25(2):282.

14. Martyn CN, Gale CR, Jespersen S, Sherriff SB: Impaired fetal growth and atherosclerosis of carotid and peripheral arteries. Lancet 1998, 352(9|23): 173.

15. Donnan PT, Thomson M, Fowkes FG, Prescott RJ, Housley E: Diet as a risk factor for peripheral arterial disease in the general population: the Edinburgh Artery Study. American Journal of Clinical Nutrition 1993, 57(6):917.

16. Lowe GD, Fowkes FG, Dawes J, Donnan PT, Lennie SE, Housley E: Blood viscosity, fibrinogen, and activation of coagulation and leukocytes in peripheral arterial disease and the normal population in the Edinburgh Artery Study. Circulation 1993, 87(6): 1915 .

17. Fowkes FG, Pell JP, Donnan PT, Housley E, Lowe GD, Riemersma RA, Prescott RJ: Sex differences in susceptibility to etiologic factors for peripheral atherosclerosis. Importance of plasma fibrinogen and blood viscosity. Arteriosclero Thromb 1994, 14(6):862-8.

18. Fowkes FG, Housley E, Riemersma RA, Macintyre CC, Cawood EH, Prescott RJ, Ruckley CV: Smoking, lipids, glucose intolerance, and blood pressure as risk factors for peripheral atherosclerosis compared with ischemic heart disease in the Edinburgh Artery Study. American Journal of Epidemiology 1992, 135(4):331.

19. Fujimoto WY, Leonetti DL, Kinyoun JL, Shuman WP, Stolov WC, Wahl PW: Prevalence of complications among second-generation Japanese-American men with diabetes, impaired glucose tolerance, or normal glucose tolerance. Diabetes 1987, 36:730.

20. Schroll M, Munck O: Estimation of peripheral arteriosclerotic disease by ankle blood pressure measurements in a population study of 60 year old men and women. J Chronic Dis 1981, 34:261.

21. Gofin R, Kark JD, Friedlander Y, Lewis BS, Witt H, Stein Y, Gotsman MS: Peripheral vascular disease in a middle-aged population sample: The Jerusalem Lipid Research Clinic Prevalence Study. Isr J Med Sci 1987, 23:157.

22. Allan PL, Mowbray PI, Lee AJ, Fowkes FG: Relationship between carotid intima-media thickness and symptomatic and asymptomatic peripheral arterial disease. The Edinburgh Artery Study. Stroke 1997, 28(2):348.

23. Meijer WT, Hoes AW, Rutgers D, Bots ML, Hofman A, Grobbee DE: Peripheral arterial disease in the elderly: The Rotterdam Study. Arterioscler Thromb Vasc Biol 1998, I 8(2): 185-92.

24. Bots ML, Hofman A, Grobbee DE: Common carotid intimamedia thickness and lower extremity arterial atherosclerosis: The Rotterdam Study. Arteriosclerosis \& Thrombosis 1994, I4: 1885.

25. Zheng ZJ, Sharrett AR, Chambless LE, Rosamond WD, Nieto FJ, Sheps DS, Dobs A, Evans GW, Heiss G: Associations of ankle-brachial index with clinical coronary heart disease, stroke and 
preclinical carotid and popliteal atherosclerosis: the Atherosclerosis Risk in Communities (ARIC) Study. Atherosclerosis I997, I 3 I(I): I I5.

26. Fowkes FG, Housley E, Cawood EH, Macintyre CC, Ruckley CV, Prescott RJ: Edinburgh Artery Study: prevalence of asymptomatic and symptomatic peripheral arterial disease in the general population. International Journal of Epidemiology 1991, 20(2):384.

27. Ogren M, Hedblad B, Isacsson SO, Janzon L, Jungquist G, Lindell SE: Non-invasively detected carotid stenosis and ischaemic heart disease in men with leg arteriosclerosis. Lancet 1993, 342: 1138.

28. Criqui MH, Denenberg JO, Langer RD, Fronek A: The epidemiology of peripheral arterial disease: importance of identifying the population at risk. Vascular Medicine 1997, 2(3):221.

29. Leng GC, Fowkes FG, Lee AJ, Dunbar J, Housley E, Ruckley CV: Use of ankle brachial pressure index to predict cardiovascular events and death: a cohort study. BMJ I996, 3 I3(7070): | 440.

30. Newman AB, Shemanski L, Manolio TA, Cushman M, Mittelmark M, Polak JF, Powe NR, Siscovick D: Ankle-arm index as a predictor of cardiovascular disease and mortality in the Cardiovascular Health Study. Arterioscler Thromb Vasc Biol I999, I 9(3):538-45.

31. Tsai AW, Folsom AR, Rosamond WD, Jones DW: Ankle-brachial index and 7-year ischemic stroke incidence: the ARIC study. Stroke 200I, 32(8): I72I.

32. Vogt MT, Cauley JA, Newman AB, Kuller LH, Hulley SB: Decreased ankle/arm blood pressure index and mortality in elderly women. JAMA 1993, 270:465

33. Nelson J]: Lower extremity arterial disease as a predictor of CHD and mortality, and the association of serum albumin with lower extremity arterial disease and other indices of atherosclerosis. Dept. of Epidemiology, University of North Carolina School of Public Health; 1996.

34. Fuller WA: Measurement error models. New York, John Wiley \& Sons; 1987.

35. Carroll RJ, Ruppert D, Stefanski LA: Measurement error in nonlinear models. London, Chapman \& Hall ; 1995.

36. Whittemore AS: Errors-in-variables regression using Stein estimates. The American Statistician 1989, 43(4):226-227.

37. The Al: The Atherosclerosis Risk in Communities (ARIC) Study: design and objectives. American Journal of Epidemiology 1989, I 29:687.

38. Higgins M, Province M, Heiss G, Eckfeldt J, Ellison RC, Folsom AR, Rao DC, Sprafka JM, Williams R: NHLBI Family Heart Study: objectives and design. Am J Epidemiol 1996, I 43( I 2): 1219

39. Jackson R, Chambless L, Yang K, et al.: Differences between respondents and non-respondents in a multi-center community-based study vary by gender and ethnicity. J Clin Epidemiol 1996, 49:|44|.

40. National Heart LBI: NHLBI Family Heart Study: manuals of procedure. 1993 [http://www.biostat.wustl.edu/fhs/]. St. Louis, Mo. , Washington University

41. National Heart LBI: ARIC study manuals of operation. 1986 [http://www.cscc.unc.edu/aric/\#]. Chapel Hill, NC, ARIC Coordinating Center, School of Public Health, University of North Carolina

42. Mundt KA, Chambless LE, Burnham CB, Heiss G: Measuring ankle systolic blood pressure: validation of the Dinamap I846 SX. Angiology 1992, 43(7):555.

43. Critikon I: DINAMAP Adult/Pediatric and Neonatal Vital Signs Monitor. Models I846 SX and I846 SX/P. Operation Manual. Tampa, FL, Critikon, Inc.; 1988.

44. Orchard TJ, Strandness DE: Assessment of peripheral vascular disease in diabetes. Report and recommendations of an international workshop sponsored by the American Diabetes Association and the American Heart Association. September 18-20, 1992, New Orleans, Louisiana. Circulation 1993, 88(2):819.

45. DiCiccio TJ, Efron B: Bootstrap confidence intervals. Palo Alto, Calif , ; 1995.

46. Bland JM, Altman DG: Statistical methods for assessing agreement between two methods of clinical measurement. Lancet 1986, I:307.

47. Ramanathan A, Conaghan PJ, Jenkinson AD, Bishop CR: Comparison of ankle-brachial pressure index measurements using an automated oscillometric device with the standard Doppler ultrasound technique. ANZJ Surg 2003, 73: 105.
48. de Graaff JC, Ubbink DT, Legemate DA, de Haan RJ, Jacobs MJHM: Interobserver and intraobserver reproducibility of peripheral blood and oxygen pressure measurements in the assessment of lower extremity arterial disease. J Vasc Surg 200I, 33:1033.

49. Fowkes FG, Housley E, Macintyre CC, Prescott RJ, Ruckley CV: Variability of ankle and brachial systolic pressures in the measurement of atherosclerotic peripheral arterial disease. J Epidemiol Community Health I988, 42(2): I28-33.

50. Chambless LE, Zhong MM, Arnett D, Folsom AR, Riley WA, Heiss G: Variability in B-mode ultrasound measurements in the Atherosclerosis Risk in Communities (ARIC) Study. Ultrasound Med Biol 1996, 22:545.

5I. Arnett DK, Chambless LE, Kim H, Evans GW, Riley W: Variability in ultrasonic measurements of arterial stiffness in the Atherosclerosis Risk in Communities study. Ultrasound in Medicine \& Biology 1999, 25(2): I75-180.

52. Chambless LE, McMahon R, Brown SA, Patsch W, Heiss G, Shen YL: Short-term intraindividual variability in lipoprotein measurements: the Atherosclerosis Risk in Communities (ARIC) Study. Am J Epidemiol 1992, I36:1069.

53. Chambless LE, McMahon R, Wu K, Folsom A, Finch A, Shen YL: Short-term intraindividual variability in hemostasis factors: the ARIC study. Ann Epidemiol 1992, 2:723.

54. Ramsey M: Blood pressure monitoring: automated oscillometric devices. J Clin Monit I99I, 7:56.

55. Whincup PH, Bruce NG, Cook DG, Shaper AG: The Dinamap I 846SX automated blood pressure recorder: comparison with the Hawksley random zero sphygmomanometer under field conditions. J Epidemiol Community Health 1992, 46: I64-I69.

56. Seventh Report of the Joint National Committee on Prevention, Detection, Evaluation, and Treatment of Hypertension (JNC 7). JAMA 2003, 289:2560-2572.

57. Resnick HE, Lindsay RS, McDermott MM, Devereux RB, Jones KL, Fabsitz RR, Howard BV: Relationship of high and low ankle brachial index to all-cause and cardiovascular disease mortality: the Strong Heart Study. Circulation 2004, I 09(6):733-739.

58. McDermott MM, Liu K, Criqui MH, Ruth K, Goff D, Saad MF, Wu C, Homma S, Sharrett AR: Ankle-brachial index and subclinical cardiac and carotid disease: the multi-ethnic study of atherosclerosis. Am J Epidemiol 2005, I 62(I):33-4I.

59. Flegal CM, Keyl PM, Nieto FJ: Differential misclassification arising from nondifferential errors in exposure measurement. American Journal of Epidemiology I991, I34( I 0): | 233-1 244.

\section{Pre-publication history}

The pre-publication history for this paper can be accessed here:

\section{http://www.biomedcentral.com/1471-2261/6/7/prepub}

Publish with Bio Med Central and every scientist can read your work free of charge

"BioMed Central will be the most significant development for disseminating the results of biomedical research in our lifetime. "

Sir Paul Nurse, Cancer Research UK

Your research papers will be:

- available free of charge to the entire biomedical community

- peer reviewed and published immediately upon acceptance

- cited in PubMed and archived on PubMed Central

- yours - you keep the copyright
BioMedcentral 